\title{
Immunonkologie "Coming of Age» - die nächste Generation der Immun-Checkpoint-Inhibitoren
}

\author{
Dirk Schadendorf ${ }^{a}$ Dirk Jäger ${ }^{b}$

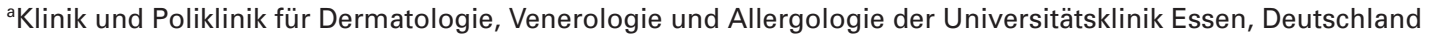 \\ ${ }^{b}$ Nationales Centrum für Tumorerkrankungen (NCT) und Universitätsklinikum Heidelberg, Medizinische Onkologie, \\ Heidelberg, Deutschland
}

Eine therapeutische Aktivierung des Immunsystems, die ein nachhaltiges Ansprechen des Tumors induzieren kann, galt Immunologen wie Onkologen bereits seit vielen Jahrzehnten als ein Hauptanliegen ihrer Forschungsbemühungen [1]. Die frühesten Ansätze zielten auf die Induktion einer Immunreaktion gegen definierte Tumorantigene mittels Vakzinierung. Den entscheidenden Proof-of-Principle lieferten jedoch die klinischen Ergebnisse, die mit dem Anti-CTLA-4-Antikörper Ipilimumab (CTLA-4 = cytotoxic T lymphocyte antigen 4 ) erzielt wurden und die erstmals eine signifikante Verlängerung des medianen Gesamtüberlebens beim nicht resezierbaren bzw. fortgeschrittenen Melanom zeigten [2]. In der zulassungsrelevanten Studie MDX010-20 erreichten vorbehandelte Patienten mit Ipilimumab ein medianes Gesamtüberleben von 10,1 gegenüber 6,4 Monaten mit einer Glykoprotein 100 (gp100)-Vakzinierung in der Kontrollgruppe $(\mathrm{p}<0,001)$. Die Therapie mit Ipilimumab ermöglichte fast einem Viertel der Patienten ein Langzeitüberleben über das Follow-up von 55 Monaten hinaus [3].

\section{Melanom als Modelltumor}

Das metastasierte Melanom gehört zu den aggressivsten soliden Tumoren und ist stark therapierefraktär. Das verbesserte molekulargenetische und tumorbiologische Verständnis, das die Identifizierung von relevanten onkogenen Treibermutationen sowie von neuen Therapiezielen im Rahmen der tumorbiologischen Immunität ermöglichte, revolutionierte die Therapiemöglichkeiten für diese Tumorentität.

Von jeher galt das Melanom als klassischer Modelltumor, da seine tumorassoziierten Antigene (TAA) auch zu den ersten Krebsantigenen überhaupt gehörten, die identifiziert und klassifiziert werden konnten [4, 5]. Einen praktischen Vorteil bietet zusätzlich die verhältnismäßig einfache Kultivierbarkeit von Melanomzellen [5]. Zudem wurden beim (zumeist noch nicht fortgeschrittenen) Melanom in Einzelfällen Spontanregressionen beschrieben, die von zellulären Entzündungsprozessen begleitet wurden und tumorinfiltrierende $\mathrm{CD}^{+}$sowie $\mathrm{CD}^{+}$T-Lymphozyten im Tumorresektat aufwiesen [6].

Die neueren immunonkologischen Therapieansätze basieren auf dem verbesserten Verständnis darüber, dass sowohl das angeborene als auch das adapative Immunsystem über die Identifizierung und Eliminierung maligner Tumorzellen an der Tumorkontrolle beteiligt sind. Zwischen dem Mikromilieu des Tumors und des Wirts spielen sich darüber hinaus immunregulative Prozesse ab, die sich auf den funktionalen Status der T-Zellen auswirken. Mittlerweile wurde die Fähigkeit von Tumorzellen, dem Immunsystem aktiv zu entgehen, als eines der charakteristischen und therapierelevanten Kennzeichen (hallmarks of cancer) des malignen Tumorwachstums mit aufgenommen [7]. Ein immuntherapeutischer Eingriff kann vor diesem Hintergrund als ein Versuch verstanden werden, die Balance zwischen Tumorwachstum und körpereigener, antitumoraler Immunantwort wiederherzustellen.

\section{Therapeutischer Paradigmenshift \\ beim metastasierten Melanom}

Indem das Immun-Checkpoint-Molekül CTLA-4 durch den Anti-CTLA-4-Antikörper Ipilimumab von der nachgeschalteten Signalkette entkoppelt wird, kann es über die aufgehobene physiologische Bremsfunktion zu einer Förderung der T-Zell-Aktivierung und einer Verstärkung tumorspezifischer Immunreaktionen kommen [8] (siehe auch «Immun-Checkpoint-Moleküle - negative Regulatoren der T-Zell-Antwort», Seiten 3-4). Mit Ipilimumab erreichte eine Patientengruppe mit fortgeschrittenem Melanom, bei der die Therapiemöglich-

\begin{tabular}{ll}
\hline KARGER & @ 2014 S. Karger GmbH, Freiburg \\
Fax +49 76145207 14 & Accessible online at: \\
Information@Karger.com & www.karger.com/ort \\
www.karger.com &
\end{tabular}


Abb. 1. Ipilimumab Langzeitverläufe: gepoolte OS-Analyse inklusive EAP-Daten (Expanded Access Program) von 4846 Melanom-Patienten (modifiziert nach [15]).

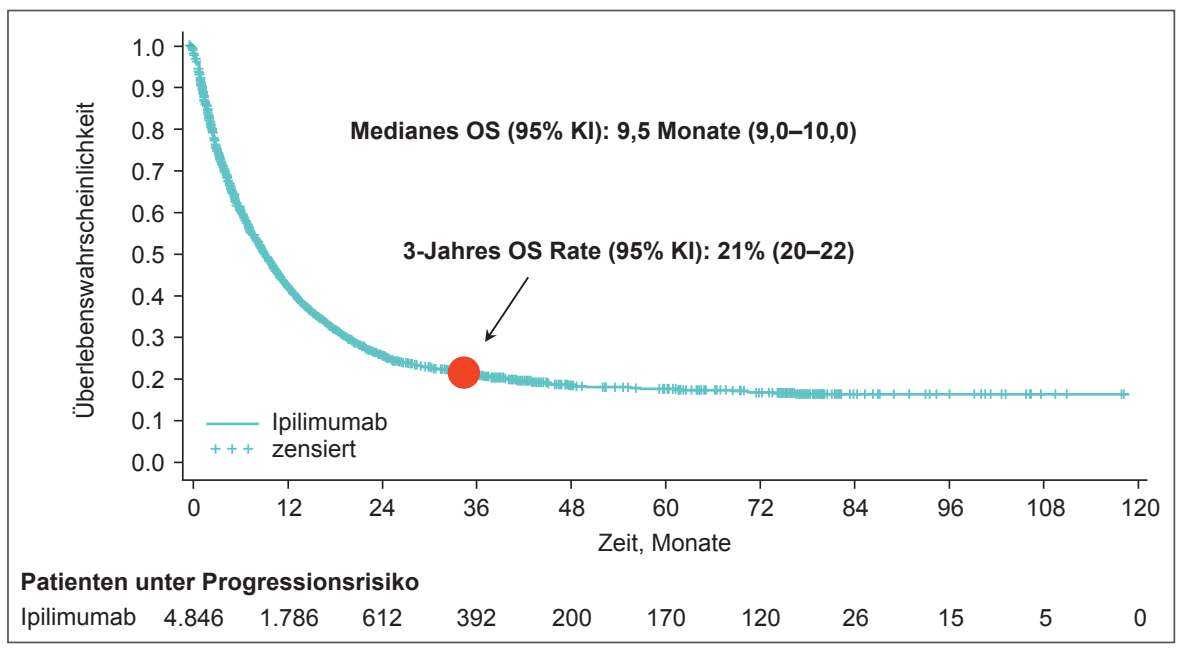

keiten bereits ausgeschöpft waren, erstmals einen statistisch signifikanten Überlebensvorteil. Dabei erwies sich vor allem das Langzeitüberleben, das die CTLA-4-Checkpoint-Blockade einem relevanten Anteil der Patienten ermöglichte, als klinisch entscheidend: Nach einem Jahr hatten 45,6 versus 25,3\% und nach einem weiteren Jahr noch 23,5 versus 13,7\% überlebt [3].

\section{Neue klinische Studienendpunkte erforderlich?}

Neben dem Potenzial, immunevasive Fähigkeiten des Tumors zu überwinden, besticht die Rationale einer ImmunCheckpoint-Inhibition somit vor allem auch durch die Aussicht auf lang anhaltende antitumorale Effekte. Sie könnten durch die Etablierung eines immunologischen Gedächtnisses sowie die Vermeidung von Resistenzentwicklungen erklärt werden, wie sie üblicherweise unter einer zielgerichteten Therapie auftreten können [9].

Allerdings werden herkömmliche Studienendpunkte der besonderen Kinetik eines immunvermittelten Ansprechens bisher nicht vollständig gerecht $[10,11]$. So kann die Therapieantwort bei einem Teil der Patienten verzögert auftreten und sogar einen initialen Pseudoprogress anzeigen, bevor es zum Rückgang des Tumors kommt. Die neu aufgetretenen Ansprechmuster wurden daher auch in die erweiterten, immunvermittelten Ansprechkriterien (immune-related response criteria, irRC) übersetzt, da sie über die herkömmlichen Kriterien der Weltgesundheitsorganisation (WHO) oder die Response Evaluation Criteria in Solid Tumors (RECIST) nicht konsequent abgedeckt werden. Dabei wird das immunvermittelte Ansprechen anhand der Änderung der Gesamttumorlast im Zeitverlauf erfasst - unter Berücksichtigung der Indexläsionen bei Baseline sowie messbarer neuer Läsionen [12].

Darüber hinaus können einige Patienten auch ohne die vollständige Eradikation des Tumors von einer langsamen Re- gression oder partiellen Response des Tumors profitieren, die in der Stabilisierung und langfristigen Krankheitskontrolle resultiert. Insbesondere die zweite Beobachtung könnte für ein neues Konzept im Sinne einer immunvermittelten «klinischen Chronifizierung» sprechen [13].

\section{Langzeitüberleben wird zum neuen Therapieziel}

Zumindest im Hinblick auf die Einordnung von Interimsdaten mit den neuen immuntherapeutischen Ansätzen sprechen die bisherigen Langzeitüberlebenskinetiken für eine Erweiterung der Bewertungsparameter: von der allein auf unmittelbare Ansprechraten bezogenen klassischen Ausrichtung klinischer Endpunkte hin zum Phänomen eines dauerhaften Langzeitansprechens, das einer längeren Nachbeobachtungszeit bedarf. Wie die Kaplan-Meier-Überlebenskurven aus der zulassungsrelevanten Studie zeigen, verliefen die IpilimumabTherapiearme und der Kontrollarm zunächst überlappend, um anschließend - nach einem Intervall von etwa 4 Monaten - getrennt zu verlaufen. Der später plateauartige Verlauf der Überlebenskurven repräsentiert die Langzeitüberleber, die über mehrere Jahre rezidivfrei blieben [15].

In der bislang größten verfügbaren Überlebenszeitanalyse von Langzeitüberlebensdaten aus 12 retro- und prospektiven Phase-II/III-Studien $(\mathrm{n}=1861)$ sowie weiteren Daten aus dem Expanded Access Program (EAP, $\mathrm{n}=2985)$ zeichnete sich der gegenüber den bisherigen Standardtherapien einzigartige Plateaueffekt in der Überlebenszeitkurve nach 3 Jahren ab: Das Gesamtüberleben zu diesem Zeitpunkt lag bei $21 \%$ (Abb. 1) [15]. Das längste dokumentierte Überleben lag bei 9,9 Jahren. Die kürzlich erfolgte Entscheidung der European Medicines Agency (EMA), die Zulassung von Ipilimumab beim fortgeschrittenen Melanom im Stadium IV auch auf die Erstlinientherapie zu erweitern, wurde auch durch diese Überlebensdaten gestützt. 
Abb. 2. Potenzielle Synergieeffekte: Hypothetisches Potenzial der Immun-Checkpoint-Blockade in Kombination mit anderen Therapien (modifziert nach [16]).

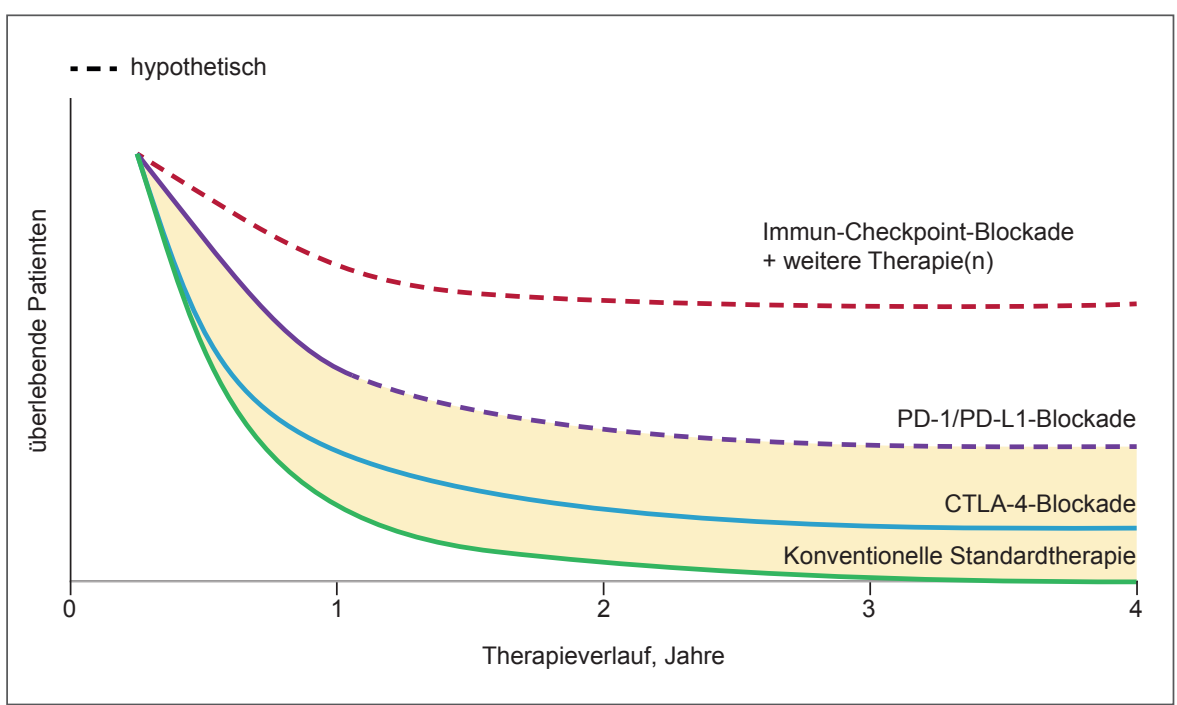

\section{PD-1-Pathway im Fokus - weitere Potenziale der immunonkologischen Therapie}

Der klinische Erfolg der Immun-Checkpoint-Blockade mit Ipilimumab hat die Entwicklung von neuen, aussichtsreichen Antikörpern vorangetrieben: So repräsentiert PD-1 (programmed cell death 1) eine weitere wichtige immunregulative Zielstruktur, die eine immunregulatorische Schlüsselfunktion ausübt (siehe auch «Immun-Checkpoint-Moleküle - negative Regulatoren der T-Zell-Antwort», Seiten 3-4). Die Bindung von PD-1 an seine Liganden PD-L1 (B7-H1) und PD-L2 (B7-DC) findet in erster Linie im peripheren Gewebe statt einschließlich des Mikromilieus eines Tumors [16] - und nicht wie bei CTLA-4 während der frühen Aktivierungsphase im lymphatischen Gewebe. Daraus könnten bei einer anti-PD1-gerichteten Therapie höhere und raschere immunvermittelte Ansprechraten sowie niedrigere Raten immunvermittelter Nebenwirkungen resultieren [17].

Derzeit befinden sich mehrere Antikörper in Entwicklung, die sich gegen PD-1 bzw. dessen Hauptliganden PD-L1 richten und deren Einsatz, ähnlich wie bei Ipilimumab, unabhängig vom Mutationsstatus infrage kommt. Erste frühe PhaseI-Daten deuten darauf hin, dass neben Melanompatienten im metastasierten Stadium künftig auch Patienten mit anderen Tumorentitäten, wie z.B. dem nichtkleinzelligen Lungenkarzinom (NSCLC) oder dem Nierenzellkarzinom (RCC), von einem immunvermittelten Tumoransprechen im Rahmen einer gegen PD-1/PD-L1 gerichteten Blockade profitieren könnten [18, 19].

Basierend auf den Daten von Phase-I/II-Studien werden nun beim Melanom gegen PD-1-gerichtete Antikörper in zahlreichen Phase-III-Studien in der Erst- und Zweitlinientherapie evaluiert (z.B. CA209-067, MK-3475-06) [18, 19]. Kürzlich wurde eine Phase-III-Studie, in der therapienaive,
BRAF-negative Patienten mit einem Stadium-III/IV-Melanom auf eine Therapie entweder mit Nivolumab oder mit Dacarbazin randomisiert worden waren $(n=418$, CheckMate066-Studie), vorzeitig abgebrochen, da die Überlegenheit der Checkpoint-Inhibition bereits vor dem geplanten Studienende durch ein unabhängiges Datenüberwachungskomitee (Data Monitoring Committee, DMC) festgestellt wurde [20]. Beim diesjährigen American Society of Clinical Oncology (ASCO)-Kongress wurden weitere Phase-I-Daten zum Langzeitansprechen beim Melanom mit dem Anti-PD-1-Antikörper Nivolumab vorgestellt, darunter auch Daten zum BRAFMutationsstatus und dem Einsatz von Ipilimumab [21, 22].

\section{Suche nach Biomarkern}

Sowohl für die Therapie mit Ipilimumab als auch für die mit Anti-PD-1-Antikörpern werden Biomarker und Selektionsmarker aktuell intensiv erforscht. Es wird diskutiert, ob die Expression des Liganden für PD-1 auf der Oberfläche von Tumorzellen beim Einsatz von PD-1-Checkpoint-Inhibitoren als prädiktiver Biomarker fungieren könnte. Da PD-L1 induzierbar ist, kann die Oberflächenexpression im Rahmen der dynamischen Immunreaktionen je nach Zeitpunkt der Messung unterschiedlich hoch ausfallen. Das heißt, dass die Expression lediglich eine Assoziation zeigt, aber vermutlich nicht als Ausschlusskriterium dienen kann, da es auch Hinweise gibt, dass per definitionem PD-L1-negative Tumore auf eine PD-1-Blockade ansprechen können [23, 24].

Das immunologische Milieu im Tumor ist sehr komplex reguliert. Daher könnten in der präziseren Charakterisierung des Tumormikromilieus (z.B. immunsuppressive Signale vs. potenzielle Immunreaktionen durch tumorinfiltrierende Lymphozyten (TIL)) und der Bestimmung der individuellen Balance zwischen Tumorprogredienz und Immunkontrolle Ansätze für die Identifizierung prädiktiver Marker liegen [25]. 


\section{Maximierung des klinischen Nutzens: \\ Rationale für Kombinationsansätze}

Strategisch ist die Evaluation von unterschiedlichen Kombinationsansätzen sinnvoll, da Tumoren sich multipler Mechanismen bedienen können, um antitumorale Immunreaktionen auszuschalten. Das Spektrum der potenziellen Kombinationspartner für die immunologische Checkpoint-Blockade reicht von weiteren Immunmodulatoren und Vakzinen über Radiatio und Chemotherapien bis hin zu Antiangiogenese bzw. zielgerichteten (targeted) Therapieansätzen. Dabei verfügen konventionelle Krebstherapien wie die Chemotherapie oder Radiotherapie ihrerseits über Wirkmechanismen, die zur Potenzierung der antitumoralen Immunantwort beitragen können [26, 27]. Beispielsweise kann ionisierende Strahlung eine vermehrte Expression von Zelloberflächenproteinen wie z.B. Klasse-I-MHC-Molekülen bewirken und den Tumor dadurch anfälliger für den Angriff zytotoxischer T-Zellen machen $[28,29]$.
Auf lange Sicht sind auch kombinierte Ansätze für ImmunCheckpoint-Modifier denkbar (Abb. 2): Die ersten Phase-IDaten einer Kombinationsstudie, die beim ASCO-Meeting 2013 vorgestellt wurden, gelten ihrerseits in klinischer Hinsicht als Proof-of-Concept für die Kombinierbarkeit von Ipilimumab mit einer anti-PD-1-gerichteten Therapie [30]. Erste Ergebnisse bei therapierefraktären Patienten mit einem Melanom im Stadium III oder IV $(\mathrm{n}=86)$ deuten darauf hin, dass ein synergistischer Effekt der dualen Immun-Checkpoint-Inhibtion vorliegen könnte - sowohl im Hinblick auf die Hemmung des Tumorwachstums als auch auf das Gesamtüberleben der Patienten.

\section{Disclosure Statement}

Die Autoren waren Referenten beim «4. Interdisziplinären Expertenforum», das von Bristol-Myers Squibb unterstützt wurde.

\section{Literatur}

1 McDermott DF, Atkins MB: PD-1 as a potential target in cancer therapy. Cancer Med 2013;2:662673.

2 Mellman I, Coukos G, Dranoff G: Cancer immunotherapy comes of age. Nature 2011;480:480-489.

$\checkmark 3$ Hodi FS, O'Day SJ, McDermott DF, et al.: Improved survival with ipilimumab in patients with metastatic melanoma. N Engl J Med 2010;363: 711-723.

4 Boon T, van der Bruggen P: Human tumor antigens recognized by $\mathrm{T}$ lymphocytes. J Exp Med 1996;183:725-729.

$\checkmark 5$ Houghton AN, Gold JS, Blachere NE: Immunity against cancer: lessons learned from melanoma. Curr Opin Immunol 2001;13:134-140.

6 Wenzel J, Bekisch B, Uerlich M, et al.: Type I interferon-associated recruitment of cytotoxic lymphocytes: a common mechanism in regressive melanocytic lesions. Am J Clin Pathol 2005;124: $37-48$.

7 Hannahan D, Weinberg RA: Hallmarks of cancer: the next generation. Cell 2011;144:646-674.

$>8$ Weintraub K: Releasing the brakes. Nature 2013; 504:S6-S8.

9 Wolchok J, Hodi FS, Weber JS, et al.: Development of ipilimumab: a novel immunotherapeutic approach for the treatment of advanced melanoma. Ann N Y Acad Sci 2013;1291:1-13.

10 Page DB, Postow MA, Callahan MK, et al.: Immune modulation in cancer with antibodies. Annu Rev 2014;65:185-202.

11 Ribas A, Hersey P, Middleton MR, et al.: New challenges in endpoints for drug development in advanced melanoma. Clin Cancer Res 2012;18:336341.

12 Wolchok JD, Hoos A, O’Day S, et al.: Guidelines for the evaluation of immune therapy activity in solid tumors: immune-related response criteria. Clin Cancer Res 2009;15:7412-7420.
13 Eggermont AM, Kroemer G, Zitvogel L: Immunotherapy and the concept of a clinical cure. Eur J Cancer 2013;49:2965-2967.

14 Prieto PA, Yang JC, Sherry RM, et al.: CTLA-4 blockade with ipilimumab: long-term follow-up of 177 patients with metastatic melanoma. Clin Cancer Res 2012;18:2039-2047.

15 Schadendorf D, Hodi FS, Robert C, et al.: Pooled analysis of long-term survival data from phase II and phase III trials of ipilimumab in metastatic or locally advanced, unresectable melanoma. European Cancer Congress 2013; abstr LBA24.

16 Ott PA, Hodi S, Robert C: CTLA-4 and PD-1/ PD-L1 blockade: new immunotherapeutic modalities with durable clinical benefit in melanoma patients. Clin Cancer Res 2013;19:5300-5309.

17 Robert C, Soria JC, Eggermont AMM: Drug of the year: programmed death-1 receptor/programmed death-1 ligand-1 receptor monoclonal antibodies. Eur J Cancer 2013;49:2968-2971.

18 Wolchok JD, Kluger H, Callahan MK, et al.: Nivolumab plus ipilimumab in advanced melanoma. N Engl J Med 2013;369:122-133.

19 Hamid O, Robert C, Daud A, et al.: Safety and tumor responses with lambrolizumab (anti-PD-1) in melanoma. N Engl J Med 2013;369:134-144.

20 Bristol-Myers Squibb Co.: Phase 3 first-line melanoma study of nivolumab, an investigational PD-1 checkpoint inhibitor, demonstrates superior overall survival compared to dacarbazine; study stopped early. http://news.bms.com/press-release/ phase-3-first-line-melanoma-study-nivolumab-investigational-pd-1-checkpoint-inhibitor\#sthash.0i4nqjMo.dpuf (Zugriff 06.08.2014).

21 Hodi FS, Sznol M, Kluger HM, et al.: Long-term survival of ipilimumab-naive patients (pts) with advanced melanoma (MEL) treated with nivolumab (anti-PD-1, BMS-936558, ONO-4538) in a phase I trial. J Clin Oncol 2014;32:5s(suppl):abstr 9002.
22 Sznol M, Kluger HM, Callahan MK, et al.: Survival, response duration, and activity by BRAF mutation (MT) status of nivolumab (NIVO, anti-PD-1, BMS-936558, ONO-4538) and ipilimumab (IPI) concurrent therapy in advanced melanoma (MEL). J Clin Oncol 2014;32:5(suppl):abstr LBA9003) .

23 Sharma P: PD-1/PD-L1 inhibition: identifying relevant biomarkers. ASCO 2014.

24 Brahmer JR, Horn L, Gandhi L, et al.: Nivolumab (anti-PD-1, BMS-936558, ONO-4538) in patients (pts) with advanced non-small-cell lung cancer (NSCLC): survival and clinical activity by subgroup analysis. J Clin Oncol 2014;32(suppl):abstr 8112.

25 Griewank KG, Ugurel S, Schadendorf D, Paschen A: New developments in biomarkers for melanoma. Curr Opin Oncol 2013;25:145-151.

26 Zitvogel L, Apetoh L, Ghiringhelli F, et al.: The anticancer immune response: indispensable for therapeutic success? J Clin Invest 2008;118:1991-2001.

27 Higgins JP, Bernstein MB, Hodge JW: Enhancing immune responses to tumor-associated antigens. Cancer Biol Ther 2009;8:1440-1449.

28 Chakraborty M, Abrams SI, Camphausen K, et al.: Irradiation of tumor cells upregulates Fas and enhances CTL lytic activity and CTL adoptive immunotherapy. J Immunol 2003;170:6338-6347.

29 Reits EA, Hodge JW, Herberts CA, et al.: Radiation modulates the peptide repertoire, enhances MHC class I expression, and induces successful antitumor immunotherapy. J Exp Med 2006;203: 1259-1271.

30 Wolchok JD, Kluger HM, Callahan MK, et al.: Safety and clinical activity of nivolumab (antiPD-1, BMS-936558, ONO-4538) in combination with ipilimumab in patients (pts) with advanced melanoma (MEL). J Clin Oncol 2013;31(suppl): abstr 9012 . 